\title{
Neuropsychiatric Symptoms in Mild Cognitive Impairment: A Literature Review
}

\author{
Evangelia Martin Latha Velayudhan \\ Department of Old Age Psychiatry, Institute of Psychiatry, Psychology and Neuroscience, King's College London, \\ London, UK
}

\section{Keywords}

Neuropsychiatric symptoms - Mild cognitive impairment .

Dementia $\cdot$ Mild behavioural impairment $\cdot$ Risk factors

\begin{abstract}
Background: Neuropsychiatric symptoms (NPS) in dementia have received much attention due to their high prevalence and their significant implications. NPS in mild cognitive impairment $(\mathrm{MCl})$, a clinical concept proposed as an intermediate state between normal aging and dementia, is now gradually gaining in interest. We aimed to conduct a selective review to examine the prevalence rate of NPS in $\mathrm{MCl}$ and associations of NPS symptoms with disease progression. Summary: We searched the PubMed database for articles on NPS in $\mathrm{MCl}$ and included articles that fulfilled the inclusion criteria. NPS was present in $35-85 \%$ of $\mathrm{MCl}$ patients. The most common symptoms were depression, irritability, apathy, anxiety, agitation, and sleep problems. Although the associated risk for disease progression of some symptoms, such as apathy and anxiety, was more consistent across studies, evidence was conflicting for symptoms like depression and sleep problems. NPS tend to co-occur, and certain combinations of NPS had a mutual or cumulative effect on disease progression. Late-onset NPS, even in a mild form (mild behavioural impairment) were found to be asso-
\end{abstract}

karger@karger.com

(c) 2020 S. Karger AG, Basel

www.karger.com/dem

Karger ${ }^{\prime}=$ ciated with an increased risk of dementia, even in the absence of cognitive impairment. Key Messages: NPS are highly prevalent in $\mathrm{MCl}$ patients and associated with subsequent cognitive deterioration. Late-onset NPS should raise suspicions of neurodegeneration. Future studies with improvised methodology are required to understand the interrelations of NPS and the role they play in the prognosis for patients with $\mathrm{MCl}$.

(c) 2020 S. Karger AG, Basel

\section{Introduction}

Over the past years, increasing attention has been paid to the presence of neuropsychiatric symptoms (NPS) in dementia, also known as the behavioural and psychological symptoms of dementia. These refer to non-cognitive, behavioural, or psychiatric symptoms linked to neurocognitive disease, and they involve disturbances in perception, affect, and behaviour [1]. Almost all dementia patients develop NPS at some point, which suggests that NPS represent core features of dementia [2]. NPS are of particular importance due to the associated severe complications for patients, caregivers, and society. Specifically, NPS cause significant distress to patients and increase suffering and morbidity [3]. In addition, they are associ-

Dr. Latha Velayudhan

Main IoPPN Building, King's College London

De Crespigny Park

London SE5 8AF (UK)

latha.velayudhan@kcl.ac.uk 
ated with increased functional impairments, greater dependence, and a greater burden on caregivers [4]. Finally, NPS are associated with earlier and longer institutionalization; this implies higher economic and social costs [5].

Research suggests that NPS are highly prevalent even in the prodromal stages of dementia, specifically with mild cognitive impairment (MCI) [6]. MCI reflects the transitional period between normal aging and dementia [7]. However, it is important to note that not all MCI patients progress to dementia [7]. Hence, there is a need to identify markers that predict the progression from MCI to Alzheimer disease (AD). On this note, the presence of NPS in MCI patients is associated with greater cognitive and functional impairments compared to those without NPS [8]. Furthermore, NPS in MCI patients significantly increases the risk of subsequent cognitive decline and, ultimately, dementia. This means that NPS in patients with MCI are important targets for further research for both prognostic and preventative purposes. It is therefore important to understand more about NPS in MCI patients because (1) this can enhance the chance of an early diagnosis, (2) NPS are markers of a high risk of rapid progression to dementia, and (3) management of NPS can minimize devastating consequences for patients, caregivers, and society, and lead to more successful outcomes.

The aim of this review is to examine the literature on the prevalence rate of NPS in MCI as well as the relationship between specific NPS and MCI progression to dementia.

\section{Methods}

Online literature search using PubMed Database was conducted using the keywords "mild cognitive impairment," "neuropsychiatric symptoms," "non-cognitive symptoms," "behavioural and psychological symptoms of dementia," and "dementia." In addition, the reference lists of each article retrieved were also examined manually to identify other studies. Articles were selected if they fulfilled all of the following criteria: (1) MCI patients were diagnosed by validated criteria, (2) NPS data were collected using standardized measures, and (3) articles were published in the English language and were peer-reviewed.

\section{Results and Discussion}

\section{Prevalence of NPS and Associated Risk in MCI}

As reported previously, 35-85\% of MCI patients exhibit at least one neuropsychiatric symptom $[6,9]$. Although the occurrence of NPS increases the likelihood of deterioration, not all symptoms are associated with disease progression and individual NPS therefore have different values as disease markers [10].

The study characteristics and prevalence rate of each symptom and its association with progression are detailed in Tables 1 and 2.

\section{Depression}

The most frequently reported symptom in MCI patients is depression, with a prevalence rate in the range of $20 \%$ (population-based samples [11]) to $83 \%$ (clinicbased samples [10]). Depression is the most studied neuropsychiatric symptom in MCI patients, and has been associated with cognitive impairment as well as a doubled risk of progression [12]. However, evidence is conflicting, as some studies have failed to demonstrate an association between depression and cognitive decline [13]. One reason for this controversy is the methodological differences across studies, in terms of measurements of depression and MCI. Even studies with similar instruments can have conflicting results, because the relationship between MCI and depression is itself a complex one [14]. For example, it is unclear whether depression occurs due to the underlying neurodegeneration or is merely a reaction to the perceived cognitive impairment by the MCI patient [15]. Depression can also lead to cognitive impairment, and although the symptoms of this are reversible, they can manifest as MCI [16]. Consequently, distinguishing whether cognitive impairments occur due to depression or MCI based on neuropsychological performance is challenging [17]. To be able to use depression as disease marker, future research should delineate its complex association with MCI. Studies that investigate whether the management of depression reduces the risk of developing MCI would be useful. They could also focus on how depression affects specific cognitive domains. Finally, they can make use of neuroimaging to examine the neural basis of the relationship between MCI and depression.

\section{Apathy}

Another highly prevalent neuropsychiatric manifestation in MCI is apathy, present in approximately $39.5 \%$ of MCI individuals in clinic-based samples [18] and $14.7 \%$ in population-based studies [11]. A longitudinal study found that MCI patients with apathy had a 7-fold risk of a progression to $\mathrm{AD}$ [13]. The association between apathy and subsequent decline is more consistent across studies, especially when compared to depression [19]. A longitudinal study with 397 MCI patients without major depression showed a high prevalence of apa- 


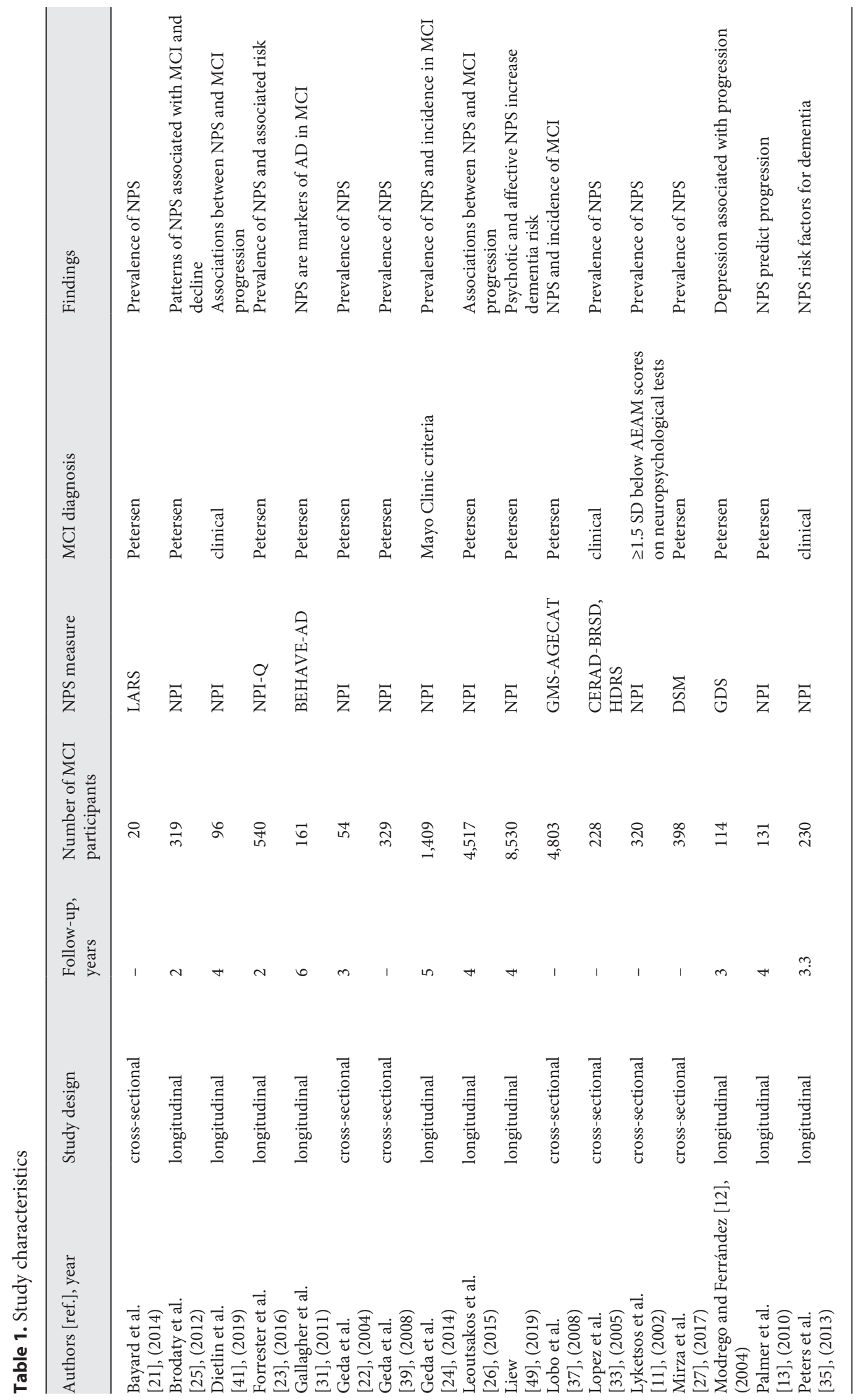




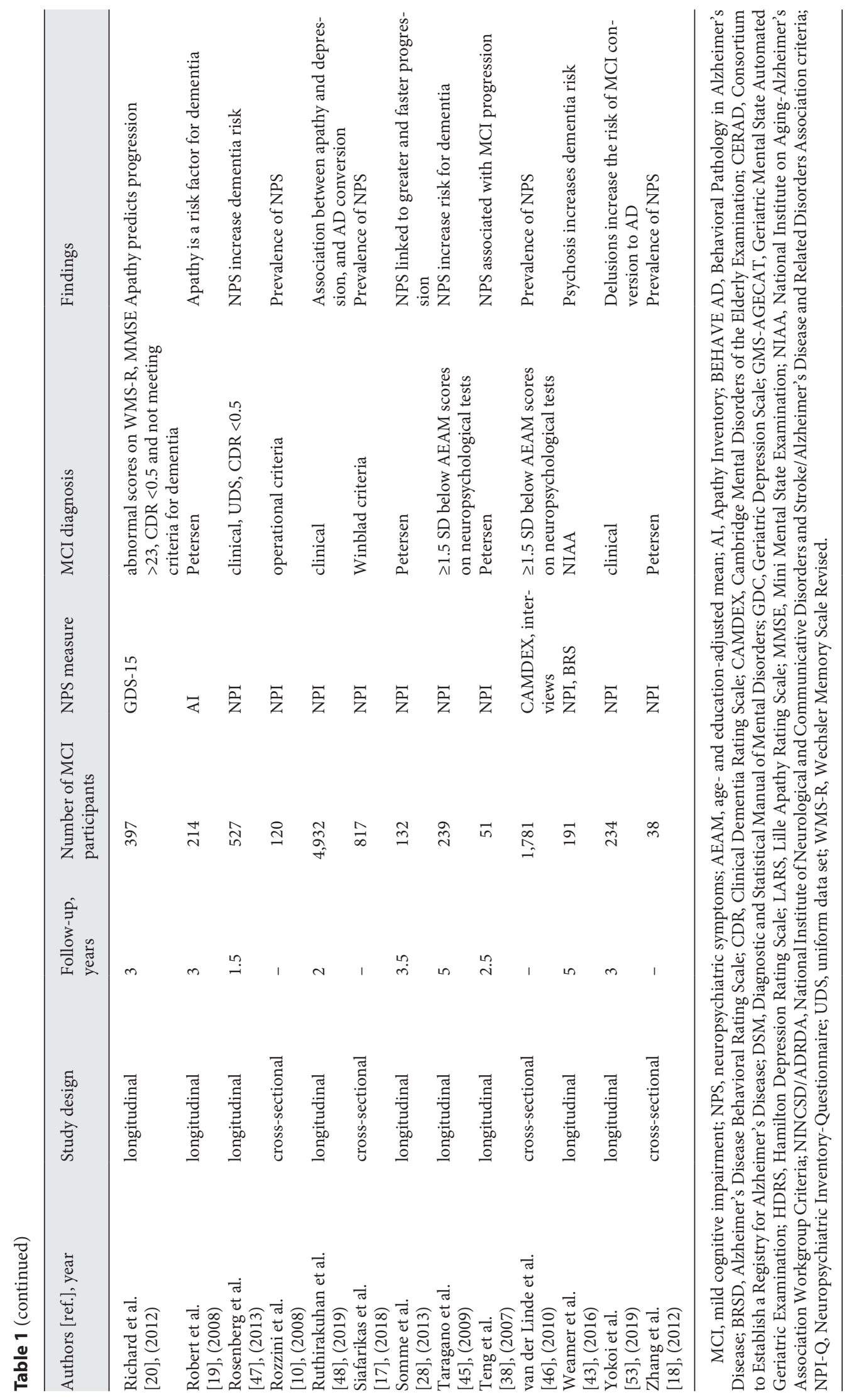


Table 2. Prevalence rate of each neuropsychiatric symptom in MCI and its association with disease progression

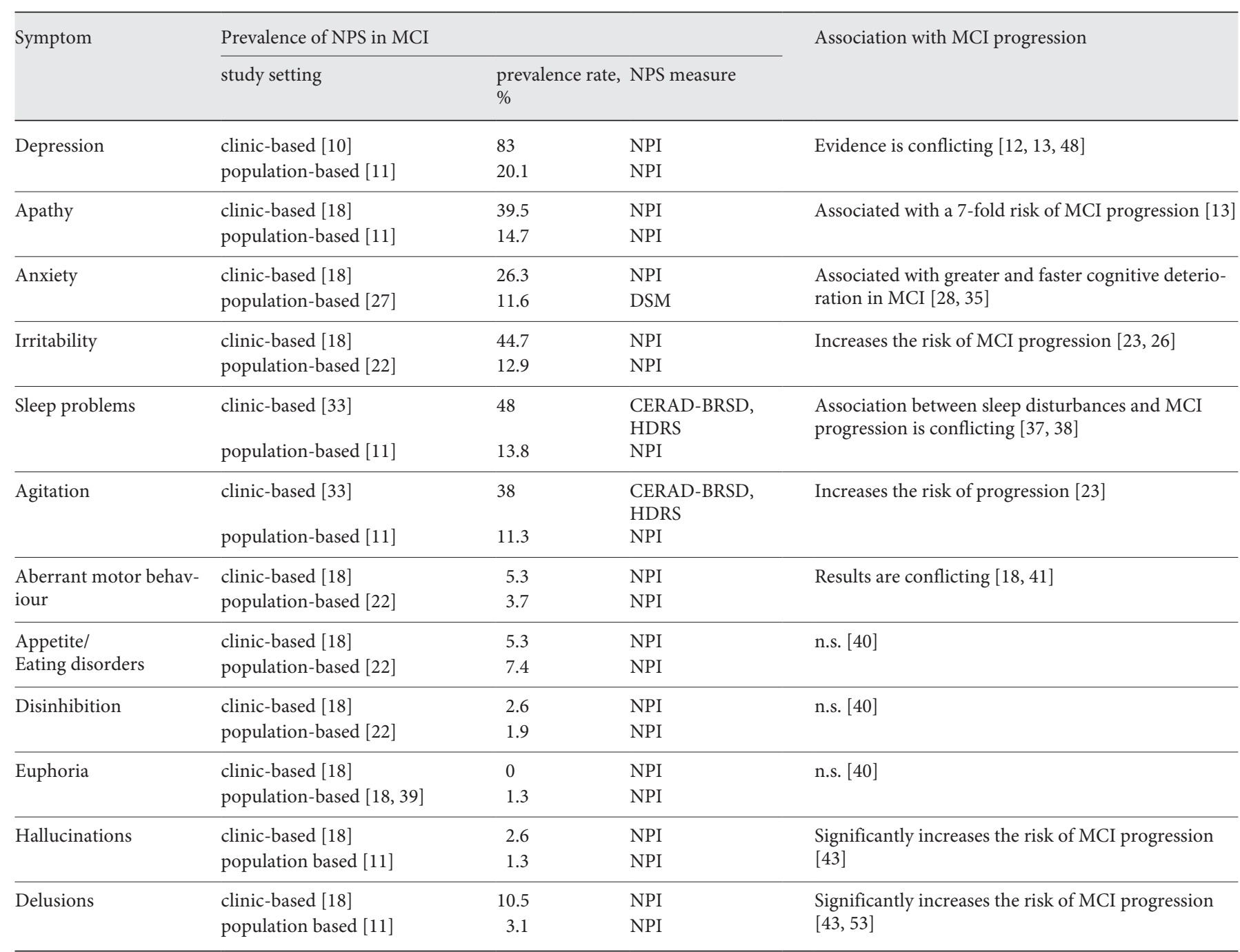

NPI, Neuropsychiatric Inventory; MCI, mild cognitive impairment; CERAD-BRSD, Consortium to Establish a Registry for Alzheimer's Disease Behaviour Rating Scale for Dementia; DSM, Diagnostic and Statistical Manual of Mental Disorders; HDRS, Hamilton Depression Rating Scale; n.s., not significant.

thy symptoms $(44.8 \%)$ and that symptoms of apathy without symptoms of depression increased the risk of the progression to AD (hazard ratio [HR] 1.85; 95\% CI 1.09-3.15) [20]. The explanation offered was that apathy in the absence of depressive affect is more strongly related to the presence of cerebrovascular disease, suggesting that there are different pathophysiological mechanisms underlying apathy and depression in MCI and dementia [20]. The high risk associated with apathy as well as its high prevalence in MCI patients make it a good marker of disease progression [20]. The association between apathy and MCI could be attributed to the fact that apathy interferes with several cognitive processes. MCI patients with apathy performed worse in executive function tests than patients without apathy, particularly in decision-making tasks [21]. Possibly, executive dysfunction associated with apathy prevents MCI individuals using compensatory ways to retain information, which can interfere with memory functioning [21]. What remains to be explored is whether apathy has a causal relationship with cognitive impairment, is a symptom of neurodegeneration, is a reaction to cognitive impairment, or is based on a shared risk factor. 
Irritability

Irritability is among the most common neuropsychiatric symptom in MCI, with a prevalence rate of $12.9 \%$ in population-based samples [22]. In clinic-based samples, the prevalence of irritability is as high as $44.7 \%$ [18]. Irritability has been associated with accelerated levels of cognitive deterioration in MCI patients [23]. It has been found that baseline levels of irritability are strongly associated with the incidence of MCI, suggesting that irritability may precede the onset of cognitive impairments [24]. However, another study found that cognitive deficits in MCI were associated with the total NPS score, but not with irritability as an individual symptom [25]; this could be attributed to the low frequency of irritability found in that particular sample. Nevertheless, other studies have found that irritability has a conversion risk similar to that of apathy [26]. These results suggest that late-onset irritability should be closely monitored in clinics, as it may indicate cognitive deterioration.

\section{Anxiety}

Anxiety is a frequently reported neuropsychiatric symptom in MCI, with a prevalence rate of $26.3 \%$ in clinical samples [18] and approximately $11.6 \%$ in populationbased samples [27]. A longitudinal study found that anxiety was one of the symptoms that significantly increased the risk of conversion from MCI to dementia and predicted a faster progression rate [28]. The interaction between anxiety and MCI can be understood in several ways. First, anxiety in MCI has been associated with impairments in global cognition and episodic and verbal memory as well as executive dysfunction [29]. Indeed, excessive worrying competes and interferes with other cognitive resources, ultimately producing these cognitive symptoms [30]. On the other hand, the symptoms of anxiety become more severe along with the increasing cognitive impairment in MCI patients [31]. Indeed, the likelihood exists that MCI patients experience anxiety due to their awareness of their cognitive impairment and their fear of further decline [32]. Thus, it is unclear whether anxiety causes or results from cognitive impairment. These 2 possibilities could occur simultaneously, making the relationship between MCI and anxiety a circular one.

\section{Agitation}

Agitation is common in MCI patients, but less common than other NPS including depression, apathy, anxiety, and irritability. Its frequency is in the range of $11.3 \%$ (population-based samples) to $38 \%$ (clinic-based sam- ples) $[11,33]$. Agitation has been associated with greater memory impairment and deficits in visuospatial abilities in MCI individuals [25]. Furthermore, a longitudinal study suggested that agitation is among the NPS associated with a 2-fold risk of cognitive decline in MCI [23]. In addition, it was found that agitated behaviours emerge in individuals before the onset of MCI, providing further evidence of an association of agitation with cognitive decline [24]. Evidence seems to support the applicability of agitation as a marker of disease progression.

\section{Sleep Disturbances}

Sleep disturbances are another highly prevalent symptom in MCI patients, varying between $13.8 \%$ in a population-based samples [11] and 48\% in clinical samples [33]. Despite their prevalence, sleep disturbances in MCI have received less attention than other NPS [34]. Although depression, irritability, and apathy are more frequent than night-time problems, sleep disturbances have been found to be the most clinically significant symptom [11]. Behaviours include difficulty falling asleep, early morning waking, and wandering during the night, all of which are risk factors for all-cause dementia and AD in a longitudinal study [35]. The evidence suggests that night-time disturbances and excessive day-time sleepiness are associated with slower reaction times and impairments of attention and memory, and thus interfere with the cognitive performance of MCI patients in neuropsychological tests [36]. Given that the MCI diagnosis relies heavily on the neuropsychological test performance, assessing and understanding the contribution of potential night-time problems is important. In addition, sleep disturbances have been found to increase the likelihood of disease progression [23,37], although some results do not support this association [38]. A possible reason for this discrepancy is that sleep disturbances are highly comorbid with other NPS such as anxiety and depression. Consequently, there is a possibility that the association between sleep problems and disease progression in MCI patients could be attributed to the neuropsychiatric comorbidity, rather than to the sleep problems as such. This requires further clarification in future studies.

Disinhibition, Euphoria, Appetite/Eating Disorders, and Aberrant Motor Behaviour

Less commonly observed NPS in MCI patients include disinhibition (1.9-2.6\%) [18, 39], euphoria (0-1.3\%) [11, $18]$, and appetite/eating disorders $(5.3-7.4 \%)[18,39]$. However, their prevalence is not significantly different from those found in cognitively intact older adults [18]. 
Moreover, in most studies, none of these symptoms has been associated with the incidence of MCI or its progression [40]. Consequently, the applicability of these NPS as predictors of cognitive deterioration is limited. Aberrant motor behaviour is also less prevalent in MCI patients (3.7-5.3\%). Nevertheless, a recent study found that aberrant motor behaviour predicted the conversion of MCI to probable $\mathrm{AD}$ after a 4-year follow-up period [41]. However, this particular study lacked statistical power due to its small sample size, and more studies are warranted to clarify the risk associated with aberrant motor behaviour in larger samples.

\section{Psychosis}

Psychotic symptoms, such as hallucinations and delusions, are rare in MCI patients. Specifically, hallucinations are present in $1.3-2.6 \%$ of MCI patients [11, 18], and delusions have a prevalence rate of $3.1-10.5 \%$ [11, 18]. Psychotic symptoms are associated with lower MMSE scores and poorer outcomes in MCI patients, compared to non-psychotic patients [42]. Psychotic symptoms represent a significant risk factor for deterioration, as MCI patients with psychosis have an 8- to 11 -fold increased risk of progressing to dementia [6,9]. Hallucinations were found to be a risk factor for vascular dementia in a longitudinal study [35]. Additionally, psychotic symptoms predict long-term cognitive status and are associated with a more rapid disease progression [43]. The high risk associated with psychosis is consistent across studies, and consequently, psychotic symptoms have a greater prognostic value than other, more frequent NPS that may not be specific to neurodegeneration [44]. However, due to the low prevalence of psychotic symptoms in MCI, they have low sensitivity in detecting at-risk individuals.

\section{Interrelations of NPS}

NPS play a significant role in MCI and the presence of $>1$ of them is associated with greater impairment and a faster decline in MCI patients [45]. However, when it comes to associations between specific NPS and progression to dementia, this becomes less clear as evidence is conflicting. This controversy around specific NPS could be attributed to the fact that many NPS tend to co-occur and overlap, and their mutual effect is not taken into account. For instance, depression in MCI has been associated with development of dementia [46], but when apathy was included in the model, depression was found to be an insignificant predictor of dementia [13]. This means that the association between depression and an increased risk of progression in MCI patients could reflect the presence of apathy, rather than depression per se [46]. In another study, the coexistence of depression and anxiety in MCI patients was associated with a more rapid cognitive decline and a higher conversion risk, compared to each symptom individually $[47,48]$. This shows that NPS can interact with each other and increase the rate of progression. These results highlight the importance of examining the relationship among various NPS and their mutual effect in progression.

When co-occurring NPS were grouped into 3 clusters, i.e., the hyperactivity (including agitation, irritability, and disinhibition), affective (including depression, apathy, sleep, anxiety, and appetite-related behaviour), and psychotic (delusions and hallucinations) clusters, all 3 clusters were associated with an increased risk of dementia [49]. However, when they were studied concurrently in the same model, only the affective and the psychotic clusters were associated with a high risk of progression [49]. In contrast, another recent study found that increased risk of MCI progression was associated with the hyperactivity and the psychotic clusters, but not with the affective cluster [41]. However, this could be due to the different methods of clustering NPS, i.e., mathematical modelinggenerated clusters [49] versus sub-syndromes proposed for $\mathrm{AD}$ [41]. This highlights the need for a consensus about the definition of NPS clusters. It is also suggested that clusters reflect NPS with shared pathophysiological changes due to neurodegeneration [50].

\section{Mild Behavioural Impairment}

A subgroup of the Alzheimer's Association, the International Society to Advance Alzheimer's Research and Treatment (ISTAART), developed the concept of mild behavioural impairment (MBI) to capture late-onset behavioural changes that do not meet the criteria for dementia or other major psychiatric conditions [15]. Thus, similar to MCI, MBI was developed in order to detect people with late-onset NPS who are at a high risk of cognitive decline and dementia. The NPS included in the MBI diagnosis involve decreased motivation, social inappropriateness, impulse control dysfunction, affective disturbances, and abnormal perception and thoughts [51].

The most widely used method to capture NPS is the Neuropsychiatric Inventory (NPI), which measures the severity and frequency of 12 behavioural symptoms: delusions, hallucinations, agitation, depression, anxiety, elation, apathy, disinhibition, irritability, aberrant motor behaviours, sleep problems, and appetite/eating disturbances [52]. The NPI does not capture all MBI domains, however, and is not tailored to predementia states [53]. 
For this reason, the MBI-C rating scale was created as an instrument to operationalize the new MBI criteria in preclinical populations [15]. To incorporate the use of MBI$\mathrm{C}$ in routine clinical assessments, future studies need to examine its reliability, validity, and utility as an instrument.

The emerging concept of MBI allows for a more explicit understanding of the relationship between NPS and MCI. A longitudinal study which followed up MBI and MCI patients for 5 years demonstrated that the presence of NPS in both groups was associated with a more rapid decline [45]. It found that MBI patients progressed to dementia, even in the absence of cognitive deficits, more rapidly than MCI patients without NPS. It also found that the MCI patients with NPS and the MBI patients with cognitive deficits progressed to dementia at a similar rate, suggesting that they actually represent 1 group [45]. A recent study found that, of the MBI domains, abnormal thoughts and perception were risk factors for dementia [53]. MBI is associated with cognitive deficits and a faster decline, particularly in attention and working memory in older adults without MCI or dementia [54]. These results suggest that late-onset NPS, even in a mild form (i.e., MBI), should raise suspicions of neurodegeneration [45, 53].

It has been found that, with the deterioration of cognitive functioning, NPS become more severe in MCI patients [31]. Moreover, the parallel progression of NPS with cognitive deficits could suggest that the underlying cause is neurodegenerational [51]. It seems that, whilst the concept of MCI incorporates the neurocognitive axis of possible prodromal dementia stages, the concept of MBI reflects the neurobehavioural axis. The 2 predementia states, namely $\mathrm{MBI}$ and $\mathrm{MCI}$, can occur simultaneously, separately, or consecutively [44]. The presence of NPS in MCI can increase the validity of MCI in predicting which individuals are more likely to progress to AD. Examining which particular symptoms are more likely to emerge in MCI patients, along with the associated risk of disease progression, will further enhance the prognostic validity of MCI.

Limitations of the review include that the search was only conducted in the PubMed database. There was significant methodological heterogeneity across studies, in terms of objectives, study setting, sample characteristics and recruitment, the assessment of both MCI and NPS, and the length of follow-up. Such methodological variability could have contributed to, at least partly, the conflicting results in the literature in terms of specific NPS, their prevalence rate, and their relationship with cogni-

Neuropsychiatric Symptoms in MCI:

A Literature Review tive impairment. Although all studies used valid measures for MCI and NPS assessment as specified by study inclusion criteria, these were not necessarily the same measures. Most of the studies used the NPI to assess NPS, but some used alternative measures (Table 1). This was particularly the case in studies that focused on individual NPS and their association with cognitive decline. For instance, the Geriatric Depression Scale and the Apathy Inventory were both used to measure apathy $[19,20]$; although these studies did not use the NPI, their results showed that apathy was an important marker of progression even when using different measurements. In terms of MCI diagnosis, most studies used the original Petersen's criteria [55], but some had other criteria (Table 1). This variability in the MCI diagnostic criteria may have contributed to variability in both the NPS prevalence rate and the relationship of NPS with cognitive deterioration. With respect to study designs and settings, the prevalence of NPS was higher in clinic-based studies than in population-based studies for all symptoms [56]. This may reflect a selection bias, as individuals referred to clinics could be at a more advanced stage of MCI, which would explain the higher NPS frequency. Furthermore, most of the data on the prevalence rate of NPS in MCI were from cross-sectional studies; this brings with it other limitations. Firstly, a cross-sectional design does not allow for a causal relationship between NPS and MCI to be established. In addition, NPS tend to fluctuate and this study design does not capture fluctuations [17]. As associations between NPS and cognitive impairment were influenced by NPS severity, the results may not be very accurate because severity also fluctuates. However, this could have been counterbalanced by the inclusion of longitudinal studies to establish the associated risks of NPS, which is a strength of this review, although the longitudinal studies had different follow-up periods, making a direct comparison between the results difficult. Progression rates are not the same for all MCI patients, which means that short follow-up periods may not be adequate to allow for MCI to progress or to capture the risks associated with NPS.

\section{Conclusions}

NPS are highly prevalent in MCI patients, with the most common ones being depression, apathy, anxiety and irritability, followed by agitation and sleep-related disorders. Euphoria, aberrant motor behavior, and disinhibition are less prevalent and, finally, psychotic symp- 
toms are rare in MCI patients. NPS may precede the onset of cognitive deficits, suggesting that late-onset behavioural changes, even in a mild form (MBI), should increase the suspicion of subsequent cognitive impairment and neurodegeneration developing. In addition, some individual NPS are associated with a greater risk of conversion from MCI to dementia. However, although the associated risk of some symptoms, such as apathy and irritability, is more consistent across studies, other symptoms, like depression, require further research as the available evidence is conflicting. Future studies need to clarify which NPS are independent risk factors, by considering the interrelations among co-occurring NPS. Furthermore, it seems that it is not clear which NPS underlie neurodegeneration or emerge as complications or reactions to cognitive deficits and/or other NPS. Future research is required to clarify this in order to enhance the prognostic ability of each individual symptom and reveal information about the underlying mechanisms in dementia.

\section{Disclosure Statement}

The authors have no conflicts of interest to declare.

\section{Funding Sources}

This research did not receive any grants from funding agencies in the public, commercial, or not-for-profit sectors.

\section{Author Contributions}

E.M. carried out the literature search and drafted the manuscript. L.V. designed the review, contributed to the literature search, and critically revised the manuscript. All authors read and approved the final version.

\section{References}

1 Mallo SC, Ismail Z, Pereiro AX, Facal D, LojoSeoane C, Campos-Magdaleno M, et al. Assessing Mild Behavioral Impairment with the Mild Behavioral Impairment-Checklist in People with Mild Cognitive Impairment. J Alzheimers Dis. 2018;66(1):83-95.

2 Steinberg M, Shao H, Zandi P, Lyketsos CG, Welsh-Bohmer KA, Norton MC, et al.; Cache County Investigators. Point and 5-year period prevalence of neuropsychiatric symptoms in dementia: the Cache County Study. Int J Geriatr Psychiatry. 2008 Feb;23(2):170-7.

3 González-Salvador T, Lyketsos CG, Baker A, Hovanec L, Roques C, Brandt J, et al. Quality of life in dementia patients in long-term care. Int J Geriatr Psychiatry. 2000 Feb;15(2):1819.

4 Lyketsos CG, Steele C, Baker L, Galik E, Kopunek S, Steinberg M, et al. Major and minor depression in Alzheimer's disease: prevalence and impact. J Neuropsychiatry Clin Neurosci. 1997;9(4):556-61.

5 Steele C, Rovner B, Chase GA, Folstein M. Psychiatric symptoms and nursing home placement of patients with Alzheimer's disease. Am J Psychiatry. 1990 Aug;147(8):104951.

6 Monastero R, Mangialasche F, Camarda C, Ercolani S, Camarda R. A systematic review of neuropsychiatric symptoms in mild cognitive impairment. J Alzheimers Dis. 2009;18(1): 11-30.

7 Petersen RC. Mild cognitive impairment as a diagnostic entity. J Intern Med. 2004 Sep; 256(3):183-94.
8 Cerejeira J, Lagarto L, Mukaetova-Ladinska EB. Behavioral and psychological symptoms of dementia. Front Neurol. 2012 May;3:73.

9 Gallagher D, Fischer CE, Iaboni A. Neuropsychiatric Symptoms in Mild Cognitive Impairment. Can J Psychiatry. 2017 Mar;62(3):161-9.

10 Rozzini L, Vicini Chilovi B, Conti M, Delrio I, Borroni B, Trabucchi M, et al. Neuropsychiatric symptoms in amnestic and nonamnestic mild cognitive impairment. Dement Geriatr Cogn Disord. 2008;25(1):32-6.

11 Lyketsos CG, Lopez O, Jones B, Fitzpatrick AL, Breitner J, DeKosky S. Prevalence of neuropsychiatric symptoms in dementia and mild cognitive impairment: results from the cardiovascular health study. JAMA. 2002 Sep; 288(12):1475-83.

12 Modrego PJ, Ferrández J. Depression in patients with mild cognitive impairment increases the risk of developing dementia of Alzheimer type: a prospective cohort study. Arch Neurol. 2004 Aug;61(8):1290-3.

13 Palmer K, Di Iulio F, Varsi AE, Gianni W, Sancesario G, Caltagirone C, et al. Neuropsychiatric predictors of progression from amnestic-mild cognitive impairment to $\mathrm{Al}$ zheimer's disease: the role of depression and apathy. J Alzheimers Dis. 2010;20(1):175-83.

14 Chan WC, Lam LC, Tam CW, Lui VW, Leung GT, Lee AT, et al. Neuropsychiatric symptoms are associated with increased risks of progression to dementia: a 2-year prospective study of 321 Chinese older persons with mild cognitive impairment. Age Ageing. 2011 Jan; 40(1):30-5.
15 Ismail Z, Agüera-Ortiz L, Brodaty H, Cieslak A, Cummings J, Fischer CE, et al.; NPS Professional Interest Area of the International Society of to Advance Alzheimer's Research and Treatment (NPS-PIA of ISTAART). The Mild Behavioral Impairment Checklist (MBIC): A Rating Scale for Neuropsychiatric Symptoms in Pre-Dementia Populations. J Alzheimers Dis. 2017;56(3):929-38.

16 Ismail Z, Elbayoumi H, Fischer CE, Hogan DB, Millikin CP, Schweizer T, et al. Prevalence of Depression in Patients with Mild Cognitive Impairment: A Systematic Review and Meta-analysis. JAMA Psychiatry. 2017 Jan;74(1):58-67.

17 Siafarikas N, Selbaek G, Fladby T, Šaltyte Benth J, Auning E, Aarsland D. Frequency and subgroups of neuropsychiatric symptoms in mild cognitive impairment and different stages of dementia in Alzheimer's disease. Int Psychogeriatr. 2018 Jan;30(1):103-13.

18 Zhang M, Wang H, Li T, Yu X. Prevalence of Neuropsychiatric Symptoms across the Declining Memory Continuum: An Observational Study in a Memory Clinic Setting. Dement Geriatr Cogn Disord Extra. 2012 Jan; 2(1):200-8.

19 Robert PH, Berr C, Volteau M, BertogliatiFileau C, Benoit M, Guerin O, et al.; PréAL Study Group. Importance of lack of interest in patients with mild cognitive impairment. Am J Geriatr Psychiatry. 2008 Sep;16(9):770-6. 
20 Richard E, Schmand B, Eikelenboom P, Yang SC, Ligthart SA, Moll van Charante EP, et al.; Alzheimer's Disease Neuroimaging Initiative. Symptoms of apathy are associated with progression from mild cognitive impairment to Alzheimer's disease in non-depressed subjects. Dement Geriatr Cogn Disord. 2012; 33(2-3):204-9.

21 Bayard S, Jacus JP, Raffard S, Gely-Nargeot MC. Apathy and emotion-based decisionmaking in amnesic mild cognitive impairment and Alzheimer's disease. Behav Neurol. 2014;2014:231469.

22 Geda YE, Smith GE, Knopman DS, Boeve BF, Tangalos EG, Ivnik RJ, et al. De novo genesis of neuropsychiatric symptoms in mild cognitive impairment (MCI). Int Psychogeriatr. 2004 Mar; 16(1):51-60.

23 Forrester SN, Gallo JJ, Smith GS, Leoutsakos JM. Patterns of Neuropsychiatric Symptoms in Mild Cognitive Impairment and Risk of Dementia. Am J Geriatr Psychiatry. 2016 Feb; 24(2):117-25.

24 Geda YE, Roberts RO, Mielke MM, Knopman DS, Christianson TJ, Pankratz VS, et al. Baseline neuropsychiatric symptoms and the risk of incident mild cognitive impairment: a population-based study. Am J Psychiatry. 2014 May;171(5):572-81.

25 Brodaty H, Heffernan M, Draper B, Reppermund S, Kochan NA, Slavin MJ, et al. Neuropsychiatric symptoms in older people with and without cognitive impairment. $\mathrm{J} \mathrm{Al}$ zheimers Dis. 2012;31(2):411-20.

26 Leoutsakos JM, Forrester SN, Lyketsos CG, Smith GS. Latent Classes of Neuropsychiatric Symptoms in NACC Controls and Conversion to Mild Cognitive Impairment or Dementia. J Alzheimers Dis. 2015;48(2):483-93.

27 Mirza SS, Ikram MA, Bos D, Mihaescu R, Hofman A, Tiemeier H. Mild cognitive impairment and risk of depression and anxiety: A population-based study. Alzheimers Dement. 2017 Feb;13(2):130-9.

28 Somme J, Fernández-Martínez M, Molano A Zarranz JJ. Neuropsychiatric symptoms in amnestic mild cognitive impairment: increased risk and faster progression to dementia. Curr Alzheimer Res. 2013 Jan;10(1):8694.

29 Beaudreau SA, O'Hara R. Late-life anxiety and cognitive impairment: a review. Am J Geriatr Psychiatry. 2008 Oct;16(10):790-803.

30 Chen C, Hu Z, Jiang Z, Zhou F. Prevalence of anxiety in patients with mild cognitive impairment: A systematic review and metaanalysis. J Affect Disord. 2018 Aug;236:21121.

31 Gallagher D, Coen R, Kilroy D, Belinski K, Bruce I, Coakley D, et al. Anxiety and behavioural disturbance as markers of prodromal Alzheimer's disease in patients with mild cognitive impairment. Int J Geriatr Psychiatry. 2011 Feb;26(2):166-72.
32 Yates JA, Clare L, Woods RT; Cognitive Func tion and Ageing Study: Wales. What is the Relationship between Health, Mood, and Mild Cognitive Impairment? J Alzheimers Dis. 2017;55(3):1183-93.

33 Lopez OL, Becker JT, Sweet RA. Non-cognitive symptoms in mild cognitive impairment subjects. Neurocase. 2005 Feb;11(1):65-71.

34 Beaulieu-Bonneau S, Hudon C. Sleep disturbances in older adults with mild cognitive impairment. Int Psychogeriatr. 2009 Aug;21(4): 654-66.

35 Peters ME, Rosenberg PB, Steinberg M, Norton MC, Welsh-Bohmer KA, Hayden KM, et al.; Cache County Investigators. Neuropsychiatric symptoms as risk factors for progression from CIND to dementia: the Cache County Study. Am J Geriatr Psychiatry. 2013 Nov;21(11):1116-24.

36 Martin JL, Ancoli-Israel S. Sleep disturbances in long-term care [vi.]. Clin Geriatr Med. 2008 Feb;24(1):39-50.

37 Lobo A, López-Antón R, de-la-Cámara C, Quintanilla MA, Campayo A, Saz P; ZARADEMP Workgroup. Non-cognitive psychopathological symptoms associated with incident mild cognitive impairment and dementia, Alzheimer's type. Neurotox Res. 2008 Oct;14(2-3):263-72.

38 Teng E, Lu PH, Cummings JL. Neuropsychiatric symptoms are associated with progression from mild cognitive impairment to $\mathrm{Al}$ zheimer's disease. Dement Geriatr Cogn Disord. 2007;24(4):253-9.

39 Geda YE, Roberts RO, Knopman DS, Petersen RC, Christianson TJ, Pankratz VS, et al. Prevalence of neuropsychiatric symptoms in mild cognitive impairment and normal cognitive aging: population-based study. Arch Gen Psychiatry. 2008 Oct;65(10):1193-8.

40 Wise EA, Rosenberg PB, Lyketsos CG, Leoutsakos JM. Time course of neuropsychiatric symptoms and cognitive diagnosis in National Alzheimer's Coordinating Centers volunteers. Alzheimers Dement (Amst). 2019 Apr; 11(1):333-9.

41 Dietlin S, Soto M, Kiyasova V, Pueyo M, de Mauleon A, Delrieu J, et al. Neuropsychiatric Symptoms and Risk of Progression to $\mathrm{Al}$ zheimer's Disease Among Mild Cognitive Impairment Subjects. J Alzheimers Dis. 2019; 70(1):25-34.

42 Steinberg M, Corcoran C, Tschanz JT, Huber C, Welsh-Bohmer K, Norton MC, et al. Risk factors for neuropsychiatric symptoms in dementia: the Cache County Study. Int J Geriatr Psychiatry. 2006 Sep;21(9):824-30.

43 Weamer EA, DeMichele-Sweet MA, Cloonan YK, Lopez OL, Sweet RA. Incident Psychosis in Subjects with Mild Cognitive Impairment or Alzheimer's Disease. J Clin Psychiatry. 2016 Dec;77(12):e1564-9.

44 Dillon C, Serrano CM, Castro D, Leguizamón PP, Heisecke SL, Taragano FE. Behavioral symptoms related to cognitive impairment. Neuropsychiatr Dis Treat. 2013;9: 1443-55.
45 Taragano FE, Allegri RF, Krupitzki H, Sarasola DR, Serrano CM, Loñ L, et al. Mild behavioral impairment and risk of dementia: a prospective cohort study of 358 patients. J Clin Psychiatry. 2009 Apr;70(4):584-92.

46 van der Linde R, Stephan BC, Matthews FE Brayne C, Savva GM; Medical Research Council Cognitive Function and Ageing Study. Behavioural and psychological symptoms in the older population without dementia-relationship with socio-demographics, health and cognition. BMC Geriatr. 2010 Nov;10(1):87.

47 Rosenberg PB, Mielke MM, Appleby BS, Oh ES, Geda YE, Lyketsos CG. The association of neuropsychiatric symptoms in MCI with incident dementia and Alzheimer disease. Am J Geriatr Psychiatry. 2013 Jul;21(7):685-95.

48 Ruthirakuhan M, Herrmann N, Vieira D, Gallagher D, Lanctôt KL. The Roles of Apathy and Depression in Predicting Alzheimer Disease: A Longitudinal Analysis in Older Adults with Mild Cognitive Impairment. Am J Geriatr Psychiatry. 2019 Aug;27(8):873-82.

49 Liew TM. Symptom Clusters of Neuropsychiatric Symptoms in Mild Cognitive Impairment and Their Comparative Risks of Dementia: A Cohort Study of 8530 Older Persons. J Am Med Dir Assoc. 2019;20(8):1054.

50 Wadsworth LP, Lorius N, Donovan NJ, Locascio JJ, Rentz DM, Johnson KA, et al. Neuropsychiatric symptoms and global functional impairment along the Alzheimer's continuum. Dement Geriatr Cogn Disord. 2012; 34(2):96-111.

51 Ismail Z, Smith EE, Geda Y, Sultzer D, Brodaty H, Smith G, et al.; ISTAART Neuropsychiatric Symptoms Professional Interest Area. Neuropsychiatric symptoms as early manifestations of emergent dementia: provisional diagnostic criteria for mild behavioral impairment. Alzheimers Dement. 2016 Feb; 12(2):195-202.

52 Cummings JL, Mega M, Gray K, RosenbergThompson S, Carusi DA, Gornbein J. The Neuropsychiatric Inventory: comprehensive assessment of psychopathology in dementia. Neurology. 1994 Dec;44(12):2308-14.

53 Yokoi Y, Takano H, Sakata M, Maruo K, Nakagome K, Matsuda H. Discrete effect of each mild behavioural impairment category on dementia conversion or cognitive decline in patients with mild cognitive impairment. Psychogeriatrics. 2019 Nov; 19(6):591-600.

54 Creese B, Brooker H, Ismail Z, Wesnes KA, Hampshire A, Khan Z, et al. Mild Behavioral Impairment as a Marker of Cognitive Decline in Cognitively Normal Older Adults. Am J Geriatr Psychiatry. 2019 Aug;27(8):823-34.

55 Petersen RC, Smith GE, Waring SC, Ivnik RJ, Tangalos EG, Kokmen E. Mild cognitive impairment: clinical characterization and outcome. Arch Neurol. 1999 Mar;56(3):303-8.

56 Apostolova LG, Cummings JL. Neuropsychiatric manifestations in mild cognitive impairment: a systematic review of the literature. Dement Geriatr Cogn Disord. 2008;25(2): $115-26$.
Neuropsychiatric Symptoms in MCI: A Literature Review
Dement Geriatr Cogn Disord 2020;49:146-155 DOI: $10.1159 / 000507078$ 